A method for selecting macro-scale structures with axially loaded members

D. Pasini ${ }^{1}$, S.C. Burgess ${ }^{2}$, D. J. Smith ${ }^{3}$

${ }^{1}$ Department of Mechanical Engineering, McGill University, Montreal, Quebec, H3A

2K6, Canada. Corresponding author contacts: email damiano.pasini@mcgill.ca, tel.514

3986295 , fax. 5143985256

2 Department of Mechanical Engineering, Bristol University, Queen's Building,

University Walk, Clifton, Bristol, BS8 1TR. U.K. email s.c.burgess@bristol.ac.uk, tel. +44 (117) 3317642 .

${ }^{3}$ Department of Mechanical Engineering, Bristol University, Queen's Building,

University Walk, Clifton, Bristol, BS8 1TR. U.K. email David.Smith@bristol.ac.uk, tel.: +44 (117) 9288075 . 


\title{
A method for selecting macro-scale structures with axially loaded members
}

\begin{abstract}
This paper presents a method to support the selection of lightweight large-scale structures. The method enables the ranking of alternative structural forms, whose axially loaded members can resist to either instability failure or material yield. Unlike previous approaches for concept design, this work models buckling failure to assess the interaction between the choice of a structural form and the choice of the cross-section shapes of its constituents. Shape transformers and scaling factors are introduced to characterize the structural efficiency of alternative crosssectional shapes. Such parameters are dimensionless and enable to measure the shape efficiency without specifying the details of the cross-section geometry. The approach eases optimization at the concept design stage and it permits to assess how the selection of the member cross-sections impacts the lightweight potential of the structural topology. The model is used to construct charts for optimizing and selecting alternative forms.

The method is applied in an industrial setting in order to compare three different structural concepts for a particular design application. The case study identified the potential performance of three structural forms and gave insight into the selection of the parameters that most influence structural performance.
\end{abstract}

KEYWORDS: lightweight design, optimization charts, performance indices, Shape Transformers, structural concept selection. 


\section{$1 \quad$ Introduction}

Mass-efficiency is often an important design goal for large-scale structures such as bulk material handling equipment, cranes and bridges. A single machine for bulk handling of iron ore or coal can weigh up to several thousand tonnes. Such a large mass leads to high material costs, high manufacturing costs and high transport costs. Low mass is also an important means for reducing environmental impact. In order to achieve low mass it is necessary to select an efficient structural form at the conceptual design stage. The selection of an efficient structural form is a challenge for a number of reasons:

- There is a wide range of different structural forms to choose from.

- There is a wide range of cross-sectional shapes and materials of constituent elements to choose from.

- Buckling failure modes must be considered in the selection process, especially for large structural forms.

Over the last century, many authors have investigated the optimum selection of structural concepts. Michell (1) was the first who set the basis of a fundamental theory for modeling optimum structural forms. In the 1960's Chan (2) and Chan (3) used the Michell theory to develop a method of graphical construction of theoretically optimal layouts. Cox (4) attempted to prove the validity of the fundamental theory for practical cases of structural design. In more recent years, Burgess (5) presented a method for ranking the efficiency of simply supported and centrally loaded structures using optimum Michell layouts as a benchmark. This was also applied to structures subjected to different load cases (6). However, in all the above research studies for concept design, failure by buckling was not modelled. 
Other related works by Cox (4), Shanley (7), Caldwell and Woodhead (8), and Ashby (9) focused on cross-sectional shape selection and material selection of a single structural element. However, these methods cannot be integrated with form selection unless all dimensions of crosssections (length, width, and thickness) are assumed to remain proportional. This limitation was overcome recently with a method that can model the efficiency of cross-sections where there is scaling in any arbitrary direction (10-12).

This paper presents a method for modeling the efficiency of large-scale structural forms where buckling is included. Whereas previous approaches for concept selection consider yield as the only failure mode (1-8), this work introduces shape transformers, scaling factors and an envelope efficiency parameter to characterize the geometric cross-section properties that govern buckling resistance. Parametric equations and design charts are developed to support the selection of structural forms and have been applied to an industrial case study. The charts give the designer insight into the interaction between cross-section selection and form selection.

\section{Research context}

This research work was carried out in collaboration with a company specializing in handling equipment for loading, unloading and storing bulk products. A main service of the company is to design and manufacture machines specialized in moving raw material from their source to the ultimate point of use via railways, ships and conveying systems. A typical example of machine is shown in Figure 1. The installations range from 300 to 6000 tonnes per hour with boom lengths up to 65 metres and bucket wheel diameter up to 10 meters. The main mass of the structure can slew on an axial bearing in order to form parallel piles on each side of the railway track. The machine shown in Figure 1 is named reclaimer because it recovers material from the bucket wheel to the central pillar. 


\subsection{Industrial requirements}

The company aimed to identify alternative concepts for the reclaimer (Figure 1) that could exploit the potential of the structural form. Besides this purpose, the industry specifies others requirements that include:

1. developing a systematic method that could model the efficiency of different pinjointed frame topologies in two dimensions.

2. including buckling failure in the modeling from the concept stage and assessing its impact on the selection of structural form concepts.

3. exploring how the structural efficiency changes with the framework size, which is specified by the max height and span of a machine.

4. discriminating the efficiency benefits provided by the form of a structure from those provided by its cross-sections.

5. providing design charts that show trends of performance in addition to the optimum values.

\subsection{Assumptions}

For modeling structural efficiency at the concept stage of design, the following simplifications were arranged with the industry:

1. The main load is assumed to be static and uniformly distributed over the horizontal boom (Figure 2). Its magnitude includes the weight of the raw material and an estimation of its own weight. On the opposite side, however, there is no restriction on the position and load distribution of the counterweight. 
2. The proposed alternatives are assumed to be pin-jointed two dimensional frameworks and to have joints with negligible weight and stiffness. The equations of equilibrium are used to estimate the internal forces.

3. The constituent members have uniform cross-section along their lengths.

4. The maximum allowable stress, $\sigma_{\mathrm{y}}$, is the same in tension and compression.

The above assumptions are used in Section 3 to formulate the structural weight for a pinjointed framework that may fail for material yield and for buckling collapse.

\section{The objective function}

Consider a pin-jointed structure consisting of $n$ members and made from a single type of material. The length of a typical member $i$ is $L_{i}$, its area is $A_{i}$ and the volume is $A_{i} L_{i}$. The total weight, $W$, of the structure is the objective function and it is given by:

$$
W=\rho g \sum_{i=1}^{n} A_{i} L_{i}
$$

where $\rho$ is the density of the material and $g$ is the gravitational constant.

Compression and tension members are included in Equation (1a), so that the structural weight is:

$$
W=\rho g\left(\sum_{i=1}^{n} A_{i}{ }^{t} L_{i}{ }^{t}+\sum_{i=1}^{n} A_{i}^{c} L_{i}^{c}\right)
$$


where $A_{i}{ }^{c}, L_{i}{ }^{c}$ and $A_{i}{ }^{t}, L_{i}{ }^{t}$ are the cross-sectional areas and lengths of the compression and tension elements respectively. We now derive the expression of the area $A_{i}^{c}$ and $A_{i}^{t}$ required to withstand the external load.

\subsection{Structural weight of frames failing for yield}

If buckling does not occur, material yield causes structure failure. This scenario has been investigated extensively in the past to contrast the lightweight potential of alternative topologies for pin-jointed frames (1-8).

The variables $A_{i}^{c}$ and $A_{i}^{t}$ of each structural member can be simply expressed in term of the load and the yield stress, and they can be substituted into Equation (1b). For tension members, the minimum area, $A_{i}{ }^{t}$, does not depend on the shape of the cross-section and it is given by:

$$
A_{i}^{t}=\frac{F_{i}^{t}}{\sigma_{y}}
$$

where $F_{i}^{t}$ is the internal force in each tension element caused by the load requirement.

Similarly, for compressive members where buckling does not govern, the minimum area, $A_{i}^{c}$, of an element does not depend on the shape of the cross-section and it is given by:

$$
A_{i}^{c}=\frac{F_{i}^{c}}{\sigma_{y}}
$$

where $F_{i}^{c}$ is the internal force in each compression member caused by the load requirement.

$$
\text { Substituting Equations (2) and (3) in (1b) gives the total weight of a framework as: }
$$




$$
W=\frac{\rho g}{\sigma_{y}}\left(\sum_{i=1}^{n} F_{i}^{t} L_{i}^{t}+\sum_{i=1}^{n} F_{i}^{c} L_{i}^{c}\right)
$$

Equation (4) confirms that the weight of a structure varies with the number of members, $n$, the forces magnitude, the member lengths as well as the material properties. Because the geometry of a cross-section is not a variable, only a change of the structural form affects the objective function. Minimizing Equation (4) provides the optima number and member lengths that yield the lightest topology.

In the past (1-6), equation (4) was used to explore the lightweight potential of alternative theoretic pin-jointed frames. However, for practical structures, especially for large-scale structures, equation (4) is of limited use, for buckling is often a common failure mode that cannot be neglected. The following section discusses such an issue and addresses a way to include instability resistance in the model.

\subsection{Structural weight for frames failing for either buckling or yield}

Large-scale structures can fail by mechanical instability before the yield compressive stress is reached. In this case, Equation (3) does not describe the minimum area for compressive member and Equation (4) does not give the correct estimation of the structural weight. The reason is that the resistance to buckling is determined by the efficiency of the cross-section and the element length. Therefore, an expression of the cross-section area is required to prevent buckling failure. This section examines models for buckling prediction and presents the approach that will be used in this work. 
The Euler formula is commonly used to model buckling failure. It is quite appropriate for long and slender struts with very small imperfections, but it gives an inaccurate prediction for practical cases. Real struts of any slenderness, indeed, collapse at buckling loads less than the Euler estimations. Improved models propose to adjust the formula with a different value of the Young's Modulus. For example, Engesser (13) suggests replacing the Young's modulus with the tangent modulus, $E_{t}$, while Karman (14) proposes to use the reduced modulus, $E_{r}$. However, both the models do not describe accurately instability. The merit of presenting an exhaustive model capable of describing the phenomenon is of Shanley (15). His theory of inelastic buckling explains that buckling starts at $E_{t}$ and reaches $E_{r}$ when the load increase causes large deformation.

Although Shanley's theory gives an accurate prediction of instability, its use can be problematic when buckling is modeled at the concept stage. The reason is that the estimation of both $E_{t}$ and $E_{r}$ requires experimental data. The former needs the strain-stress distribution to be evaluated, and the latter involves also the modeling of the convex and concave side of a strut.

Because the focus of this work is placed on the concept design, a handier model is necessary. We decide to opt for the Rankine and Gordon formula. This approach is not as accurate as that of Shanley, but it has the advantage that only material and geometric properties are needed. The formula is semi-empiric and it describes the stress caused by either buckling or yield failure for a strut of any length and with geometric imperfections. His expression, $\sigma_{f}$, represents the stability and strength constraint of a compressive member, $i$, such that:

$$
\sigma_{f}=\frac{F_{i}^{c}}{A_{i}^{c}}=\frac{\sigma_{y}}{1+\mu\left(\frac{L_{i}^{c}}{r_{g i}}\right)^{2}}
$$




$$
A_{i}^{c}=\frac{F_{i}^{c}\left(r_{g i}{ }^{2}+\mu L_{i}^{c^{2}}\right)}{\sigma_{y} r_{g i}^{2}}
$$

The key parameter in equation (6) is the radius of gyration. Once the designer specifies the cross-section geometry of a member of given length and material, $r_{g}$ can be evaluated to determine the design variable, $A_{i}^{c}$, which represents the minimum area required by each member to withstand the internal force $F_{i}^{c}$.

It is noted that $A_{i}^{c}$ is the minimum area of all compressive loaded members of a frame. However, some elements are loaded more than others and are more vulnerable to buckling. A valuable alternative to this approach where the cross-sectional area, i.e. expression (6), of each members is minimized with respect to the member stability constraint, i.e. Expression (5), involves two steps, as described in (16). The first is to impose a change of the cross section area for only the heavily loaded members. The second is to include in the problem formulation the stability constraint of the overall system.

We now recall that one key requirement of the Industrial partner is to explore how the choice of the member cross-sections impacts the selection of a topology concept. If equation (6) were used in such a form, then the designer would be forced to decide at the onset all the geometric details of a cross-section, and then calculate the value of $r_{g}$. As known, this task is left at later design stages when the details of the geometry are defined. A way to avoid such a decision is to 
provide dimensionless parameters that describe the efficiency of a cross-section and that may be substituted in equation (6). A method to do so is presented in the following section.

\section{$4 \quad$ Modelling the buckling resistance of different cross-sections}

To express the radius of gyration in term of dimensionless parameters, we resort to Shape Transformers and scaling factors (10-12). This approach has been introduced recently for comparing the relative efficiency of cross-sections in bending and for developing charts of material and shape selection. It is well suited to optimize and select concepts at the early stage of design.

The method is based on the idea that a cross-section can be described by two distinct aspects: the Envelope and the Shape. As shown in figure 3, the Envelope is the rectangle described by the cross-section size, while the Shape is the figure enclosed in it. These entities are defined to decouple the structural efficiency in two contributions: the first is governed by the Envelope scaling, and the second by the properties of the Shape.

To compare the relative efficiency of cross-sections, a reference square is introduced and the following symbols apply:

For the reference square

$B_{o}, H_{o} \quad$ width and height where $B_{o}=H_{o}$

$A_{D o}=$ area of square

$I_{D o}=$ second moment of area of square

For a generic cross-section of any Shape

$A=$ area of shape

$I=$ second moment of area 
$r_{g}=$ radius of gyration

For the Envelope of the generic cross-section:

$B, H$ : $\quad$ width and height

$A_{D}=$ area of Envelope

$I_{D}=$ second moment of area of Envelope

$r_{g D}=\quad$ radius of gyration

Expressions of area, $A$, second moment of area, $I$, and radius of gyration, $r_{g}$, are shown in Table 1 for some common cross-sections.

\subsection{Shape contribution to structural efficiency}

The Shape contribution to the cross-section efficiency is governed by a combination of Shape transformers. These are dimensionless measures of the geometric quantities of a cross-section and are analogues to the material properties, for they specify Shape properties that are invariant to any cross-section scaling.

For example, two Shape Transformers $\psi_{A}$ and $\psi_{I}$ can be introduced to describe the transformation of the area and second moment of area of a generic shape into its rectangular Envelope. They are given by:

$$
\left\{\begin{array}{l}
\psi_{I}=\frac{I}{I_{D}} \\
\psi_{A}=\frac{A}{A_{D}}
\end{array}\right.
$$


Another important properties of a cross-section geometry is the Envelope Efficiency parameter, $\lambda$, of a section. Such a measure quantifies how efficiently the Shape fills the crosssection Envelope. $\lambda$ is defined as

$$
\lambda=\frac{\psi_{I}}{\psi_{A}}=\frac{r_{g}{ }^{2}}{r_{g D}{ }^{2}}
$$

The Envelope Efficiency parameter gives a direct indication of the structural performance of different shapes for a given envelope.

We now examine expressions of the geometric quantities of cross-sections.

For rectangles

$$
\begin{aligned}
& A=A_{D} \rightarrow \psi_{A}=1 \\
& I=I_{D} \rightarrow \psi_{I}=1 \\
& r_{g}=r_{g D} \rightarrow \lambda=1
\end{aligned}
$$

for a generic cross-section

$$
\begin{aligned}
& A=\psi_{A} A_{D} \\
& I=\psi_{I} I_{D} \\
& \frac{r_{g}{ }^{2}}{{r_{g D}}^{2}}=\lambda \quad(9 \mathrm{c})
\end{aligned}
$$

Table 2 reports the shape attributes of alternative concepts, with at least one axis of symmetry. As can been seen, $\psi_{A}$ and $\psi_{I}$ characterize the area and second moment of area of a shape concept, regardless its size. Shape Transformers have been used recently to classify shapes. Families, e.g. the Ellipses and the Rectangles, and classes of shapes can describe different shapes in a way similar to material classification. When shape transformers are plotted into an efficiency map, 
members of a family cluster together and the visualized bounds help optimize design choices at different length scale (12). Each concept has a specific properties range, with values that vary with respect to the area filling the envelope. When the material fills completely the shape, we have a solid shape, and $\left(\psi_{A}, \psi_{I}\right)$ assume the range upper bounds; whereas for hollow shapes, $\left(\psi_{A}, \psi_{I}\right)$ may decrease up to zero, which corresponds to an empty Shape. For example, $\psi_{A}=\psi_{I}=1$ are the properties of a solid rectangle, the stiffest among constrained cross-sectional shapes. For hollow rectangles, $\left(0<\psi_{A}<1,0<\psi_{1}<1\right)$. Solid and hollow rectangles belong to the Rectangles family, characterized by an efficiency range $1<\lambda<3$. For other families, i.e. shape concepts, $\psi_{A}$ and $\psi_{I}$ are constants less than 1. A solid Ellipse, for instance, has always properties $\left(\psi_{A}=\pi / 4, \psi_{I}=3 \pi / 16\right)$ regardless of any Envelope scaling. For their respective hollow Ellipses, the shape transformers vary, but always within the ranges $\left(0<\psi_{A}<\pi / 4,0<\psi_{1}<3 \pi / 16\right)$.

Besides $\psi_{I}, \psi_{A}$, Table 2 lists also expressions of $\lambda$, which is the efficiency of a cross-section shape regardless its size. The ranges of $\lambda$ are given to assist early design choices. They free a designer from specifying the geometric details of a cross-section. Specific $\lambda$ thresholds permits efficiency comparison between shape concepts. The higher $\lambda$, the better the buckling resistance of the shape. These ranges, however, are theoretical because real cross-sections have reduced ranges due to the difficult task of manufacturing a material into a thin wall, shear failure requirements, and local instability. Shape transformers have been recently used to develop design charts that give insight into material and shape selection at different length scale (10-12).

\subsection{Envelope contribution to structural efficiency}

For a given type of shape such as a rectangular section, it is well known that deep sections are most efficient. This section describes a way of modeling the efficiency of different envelopes for 
a given shape. We specify a linear multiplicator, $v$, for the relative arbitrary envelope scaling between the height of the cross-section and the square, as:

$$
v=\frac{H}{H_{o}}
$$

Thus the radius of gyration, $r_{g D}$, of a generic rectangle $\left(\psi_{A=} \psi_{I=} \lambda=1\right)$ with respect to that, $r_{g D o}$, of the reference square is given by:

$$
\frac{r_{g D}^{2}}{r_{g D o}^{2}}=v^{2}
$$

Figure 4 shows how a square reference is transformed into a generic I cross-section by selecting the appropriate shape properties, $\psi_{A}$ and $\psi_{I}$. The figure shows also how $v$ describes its relative scaling in the vertical direction. Envelope scaling in other directions have been also explored (10), but this work models only vertical scaling. From Equation (10), it is clear that maximizing the multiplicator, $v$, increases the resistance to buckling of each loaded member.

\subsection{Total contribution: Shape and Envelope}

Shape Transformers and Envelope multiplicators are used in this section to express the radius of gyration in the Ranking Gordon formula.

Expression (11) is now substituted in (9c) such that the radius of gyration of a generic crosssection relative to that of the reference square is given by: 


$$
\frac{r_{g}^{2}}{r_{g D o}}=v^{2} \lambda
$$

This expression shows that $\lambda$ and $v$ describe the contributions to buckling resistance of the Shape and of the Envelope. Since the measures are dimensionless and decoupled, the designer can set the cross-section geometry without being forced to decide its real dimensions.

In the next section, Equation (12) is used in the Rankine Gordon formula to derive the crosssection area for buckling resistance.

\subsection{Cross-section area preventing buckling and yield failure}

To derive the cross-section area resisting buckling, we replace expression (12) in (6) such that:

$$
A_{i}^{c}=F_{i}^{c} \frac{\lambda v^{2} r_{g D o}^{2}+\mu L_{i}^{c^{2}}}{\sigma_{y} \lambda v^{2} r_{g D o}^{2}}
$$

where $\mu=\frac{\sigma_{y}}{\pi^{2} E}$.

For a given load, material and member length, Equation (13) allows the selection of shape properties and envelope parameter to quantify the amount of material required to avoid buckling and yield failure in each member of a pin-jointed frame.

The solution of the problem is now carried out into two steps. The first is the structural problem solved through the classic matrix formulation for a given pin-jointed framework. This requires the use of equilibrium and compatibility conditions to determine the unknown internal forces, $F_{i}^{c}$, as function of the material properties and design variables. The second one is the 
optimization problem resolved by finding the cross-section area variables that minimize the objective function. This involves minimizing the frame weight given by 1(b) with respect to the stability and strength constraint, i.e. relation (13), of each frame member. For a given concept, the result is an optimal function, which is expressed in term of the cross-section properties, that enable the search of the lightest structural form. The designer can therefore evaluate the impact that the selection of the shape transformers has on the choice of an optimum topology. This is shown in the next section for three concepts of interest to the collaborating company.

\section{$5 \quad$ Industrial case study: the alternative structural forms}

Figure 5 illustrates three structures alternatives to that shown in Figure 2. They are referred in this work as "Web", "Parallel" and "Convergent". They were selected for a number of reasons.

The Web frame is examined because it serves as the benchmark of the analysis. The benchmark defines an optimum form for a particular load so that the efficiency of the other topologies can be put into perspective. Sometimes, it can be a non-practical structure, but it has the purpose of showing that there is not a great benefit in optimizing a topology when the objective function gets close to that of the benchmark. Michell (1) demonstrated that the double cantilever illustrated in Figure (6a) is the lightest form when the failure mode of a simply supported and centrally loaded framework is yield. Figure (6b) shows the same Michell structure where the central point load and reaction are inverted (support in the central position and point loads at the end). The nesting of the inverted Michell structure (Figure 6b) is depicted in Figure (6c). The end point loads are replaced by a uniformly distributed load carried by infinite number of ties (semicircle). This theoretical structure, referred as "derived Michell frame", is the benchmark because it is the lightest form in yield design. It will be shown that for an appropriate number of ties, its performance approaches that of the Web. 
The Parallel and the Convergent concepts have been proposed for their potential. First, these forms may gain a benefit from the ties action, which is usually significant for large scale structures, such as suspended bridges. In general, ties are more efficient than beams because they have fully stressed cross-sections, while in beams there is redundant material close the neutral axis. Second, the higher the number of ties, the smaller is the bending induced in the horizontal arms, which function mainly as struts. Third, if the number of supports provided by the ties is greater than 8 , internal forces can be computed by assuming that each tension member will carry approximately half of the uniform load between two ties with exception to those at the extremities (17). Finally, the Parallel and the Convergent are forms suitable to examine how the structure weight profits by changing the height to width ratio, which is a variable of major interest to the industrial partner.

As suggested by the industry, the alternative forms shown in Figure 3 are modeled as twodimensional pin-jointed frames. The members in the third direction, i.e. out of plane, have modest buckling length and are not as unstable as those in the plane. They have a minor impact into the efficiency of the truss. For this reason, the industry decided to focus their investigation on inplane buckling, although out of plane fix-fix buckling should also be considered. Such a choice has been regarded as viable especially because the work deals with the concept stage of design. Here, the solutions may not need be accurate but rather should provide trends of performance that, although estimated, offers valuable insight to the industrial engineers.

The following sections examine the efficiency of the alternative structures by using the analysis of section 3.1 for yield design and that of section 4.4 when buckling is included. 


\subsection{Buckling not modeled}

When buckling does not occur, the structural weight is given by Equation (4). The weight of the derived Michell frame can be calculated for an unbounded number, $n$, of ties and struts and is given by:

$$
W=P L \frac{\rho g}{\sigma_{y}}
$$

Expression (14) is given for an infinite number of members and it is independent on $n$. However, for a finite number of ties $n$ and angle $\alpha$, as shown in Figure 3(a), the weight of the Web frame can be written as:

$$
W=P L \frac{\rho g}{\sigma_{y}} \frac{\pi}{2 \alpha} \tan \alpha \frac{1}{n^{2}} \sum_{i=1}^{n} i
$$

The weight of the parallel frame is given as a function of $\beta$ (Figures $3(\mathrm{~b})$ ) by:

$$
W=2 P L \frac{\rho g}{\sigma_{y}} \frac{1}{n^{2}} \sum_{i=1}^{n} i\left(\frac{2}{\tan \beta}+2 \tan \beta+\frac{2}{\sin \beta \cos \beta}\right)
$$

Similarly, the weight of the convergent frame (Figure 3(c)) is:

$$
W=2 P L \frac{\rho g}{\sigma_{y}}\left[\frac{2}{\tan \beta}+\frac{1}{n^{2}}\left(\frac{2}{n} \tan \beta \sum_{i=1}^{n} i^{2}+2 \sum_{i=1}^{n} \frac{i}{\sin \beta_{i} \cos \beta_{i}}\right)\right]
$$

where $n$ is the number of ties, $\sigma_{y}$ is the yield stress, $g$ is the gravity, $\rho$ is the density.

\section{Optimum values.}

Beta is the angle governing the overall framework size. This variable ( $\mathrm{H} / \mathrm{L}$ in Figure 3) describes the max height to width ratio of the machine and, as mentioned, it is of interest to the company. The best beta value that optimizes the structural weight is given by minimizing the expressions (16), and (17). With the following data from the industry:

$$
n=10, L=50 \mathrm{~m}, p=50 \mathrm{KN} / \mathrm{m}, \rho=7900 \mathrm{~kg} / \mathrm{m}^{3}, \sigma_{y}=300 \mathrm{MPa}, E=210 * 10^{3} \mathrm{MPa} \text {, we obtain: }
$$


Derived Michell frame $W=102 \quad \mathrm{KN}$

Web: $\quad W=112 \quad \mathrm{KN}$

Convergent: $\quad W_{\min }=160 \quad \mathrm{KN} \quad$ for $\beta=58.18^{\circ}$

Parallel: $\quad W_{\text {min }}=142 \mathrm{KN} \quad$ for $\beta=45^{\circ}$

$n=10$ is a sufficient number of ties to meet the assumptions and to get the performance of the Web closed to that of the derived Michell's frame. Therefore, the Web form is taken as the benchmark structure to compare to the Convergent and the Parallel forms.

\section{Design charts}

Design charts are used to examine performance trends, one of the goal of the industrial partner. Equations (14), (15), (16), and (17) are plotted as function of $\beta$ to explore how the structural weight changes with the size of the machine. The results are shown in Figure 5.

Among real structures, the web structure is the best for yield design because it is derived from the Michell layout. The performances of Parallel and Convergent frames are quite close. Although the convergent can provide a minimum lower than that of the parallel, the latter performs better for beta angle greater than $60^{\circ}$. The chart shows also that the convergent is less sensitive to variation of the size machine.

In this scenario, the members are assumed to be always stable for buckling and the best structural form does not depend on the selection of the cross-section. This result is consistent to that obtained with previous methods for concept form selection (1-6). However, they were of limited use for the industry involved in this case study. 


\subsection{Buckling considered}

When buckling is taken into account, Equation (13) describes the stability and strength constraint to be used to solve the optimization problem. This section examines the three alternative forms for different cross-section efficiencies. We use the data from the company to assess the impact of buckling and to obtain insight into how the cross-section selection may affect the selection of a structural form.

\section{Optimum values}

Although a number of cross-section efficiencies were examined, this work presents, as an example, the results of four cases. The first two (Figures 8 and 9) describe cases where the designer decides to keep the cross-section size unaltered and assesses the impact of a shape change. As a result the efficiency of the topology is affected only by the shape efficiency parameter $\lambda$. On the contrary, the other two cases (Figures 10 and 11) represent scenarios where the designer does not attempt to minimize weight by optimizing the shape; rather he chooses to scale the envelope. Hence the shape efficiency contribution remains constant and the envelope contribution is the only one governing relative weight variation. The following report the four cases.

1) We start with solid circular cross-sections that have shape efficiency of $\lambda=3 / 4$ (see Table 2). The minimum structural weight and the optimum values of the variable $\beta$ are:

$\begin{array}{llll}\text { Web: } & W=886 & \mathrm{KN} & \\ \text { Convergent: } & W_{\text {min }}=1876 & \mathrm{KN} & \text { for } \beta=62.64^{\circ} \\ \text { Parallel: } & W_{\text {min }}=298 & \mathrm{KN} & \text { for } \beta=54.88^{\circ}\end{array}$


2) Then we select a different shape, i.e. hollow circular cross-section, that still belong to the Ellipses family. From Table 2, the shape efficiency contribution for this family should be in the range $3 / 4<\lambda<9 / 4$. We chose $\lambda=2$ and we do not impose any scaling of the envelope with respect to the previous case. Thus, the minimum structural weight and the optimum values of the variable $\beta$ are given by:

Web: $\quad W=489 \quad \mathrm{KN}$

Convergent: $\quad W_{\min }=931 \quad \mathrm{KN} \quad$ for $\beta=58.7^{\circ}$

Parallel: $\quad W_{\min }=189 \mathrm{KN} \quad$ for $\beta=51.1^{\circ}$

3) In this scenario, we change shape properties as well impose envelope scaling. An hollow rectangular cross-section with shape efficiency of $\lambda=2.3$ is chosen. The envelope scaling is imposed to be $v=0.6$. Here, the minimum structural weight and the optimum values of the variable $\beta$ are:

$$
\begin{array}{llll}
\text { Web: } & W=586 & \mathrm{KN} & \\
\text { Convergent: } & W_{\text {min }}=1170 \mathrm{KN} & \text { for } \beta=60.4^{\circ} \\
\text { Parallel: } & W_{\text {min }}=216 & \mathrm{KN} & \text { for } \beta=52.5^{\circ}
\end{array}
$$

4) Finally, a hollow rectangular cross-sections with the same shape efficiency, i.e. $\lambda=2.3$, of case 3 is scaled with $v=1.1$. In this case, the structural weight and the optimum values of the variable $\beta$ are given by: 


$$
\begin{array}{llll}
\text { Web: } & W=440 & \mathrm{KN} & \\
\text { Convergent: } & W_{\text {min }}=804 & \mathrm{KN} & \text { for } \beta=57.2^{\circ} \\
\text { Parallel: } & W_{\text {min }}=175 & \mathrm{KN} & \text { for } \beta=50^{\circ}
\end{array}
$$

Comparing the above values with those obtained in Section 4.1 shows that it is essential to include the modeling of buckling at the concept stage. Buckling lowers failure resistance and extra material is required to prevent failure. As expected, the impact on the efficiency is substantial. The weight can double that obtained for yield failure in the best scenario. But in the worse, it can be up to 18 times.

\section{Design charts}

This section examines the weight trends for the alternative structural forms resisting buckling. The plots are shown in Figures 8, 9, 10, and 11, and are given in term of shape and envelope contributions for the cross-section efficiencies considered in this work. These are shown at the top of each chart. A number of insight can be drawn by inspecting the charts.

When the curves are compared to those obtained for yield failure (Figure 7), it can be seen that the ranking of the structural form has changed and there is a considerable effect on the selection of the best form. The Web is not the lightest structure concept because it has numerous struts required to withstand buckling. This feature impacts also the general performance trends of the concepts. The curves in buckling design are afar one from another and their relative position is not as close as that obtained for yield design.

The convergent frame performs always worse because the central pillar has to support a constant compressive force along the whole length. This does not occur in the parallel frame where the height decrease of each strut pair lowers the vertical load. Furthermore, the parallel is 
to be preferred because its performance is less sensitive to variation of $\beta$. This feature is significant because gives the designer the freedom to accommodate constraints that may limit the design space.

Other insight can be drawn by inspection of the charts in Figures 8-11. Reductions in weight are obtained by using the efficiency range shown in Table 2 for different shape concepts. For example, the plain comment that hollow sections perform generally better than solid crosssections for a given envelope can be obtained when the trends in Figure 8 are contrasted to those in Figure 9. However, the benefit differs for each typology. The convergent form makes the most of the selection of hollow cross-section, because the weight reduction is $50 \%$ of that obtained with solid cross-section. The same shape change applied to the Web and Parallel typologies, however, yield to a lower performance benefits, i.e. $45 \%$ and $37 \%$ respectively. On the other hand, when hollow shapes are chosen to provide the same efficiency, as the hollow rectangular cross-sections illustrated in Figures 10 and 11, the designer can still obtain $20 \%$ of weight variation by the sole envelope contribution. This confirms that decoupling the shape contribution from that of the envelope gives designers the freedom to decide whether the lightest typology is obtained by optimizing either the shape or the size or both.

Besides the impact that the selection of the cross-section has on the choice of a structural form, the case study has shown also that the effect of ties is beneficial for large scale structures. However, only a proper selection of the Shape and Envelope parameters can exploit the benefit of a structural form.

\section{$6 \quad$ Concluding remarks}

Unlike previous methods of form selection for the early stage of design, this paper has presented a method for modelling the mass-efficiency of large-scale structures where buckling is included as a failure mode. The method models the geometric properties of arbitrary scaled cross- 
sections and defines dimensionless efficiency factors. These are included in parametric equations that are used at the concept stage in order to provide insight into the efficiency of a structural concept. Design charts have shown that the interaction between structural form and cross-sections of compressive members affects the selection of the best structure. The method has been carried out on an industrial case study in order to compare three different structural forms for a particular design application. The case study identified the potential of the three structural forms and gave insight into the selection of the parameters that optimize structural efficiency.

\section{$7 \quad$ Acknowledgements}

The authors would like to acknowledge the support of Eng. Tony Burnett of Metso Minerals Industries Inc., for his support and comments on this work.

\section{$8 \quad$ References}

1. Michell A. G. M., (1904), "The limits of economy of material in frame-structures", Phil. Mag., 8, 589-597.

2. Chan A.S.L., (1960), "The Design of Michell Optimum Structures", The college of Aeronautics Cranfield Report 142.

3. Chan H.S.Y., (1963), "Optimum Michell Frameworks for Three Parallel forces", The College of Aeronautics, Cranfield Report 167.

4. Cox H.L., (1965), The design of structures of least weight, Oxford Pergamon Press.

5. Burgess S.C., (1998), "The ranking of efficiency of structural layouts using form factors", Journal of Engineering Science, 212, 117-128.

6. Burgess S.C., Pasini D., Smith D.J., (2001), "Form factors: a design method to support the selection of structural concepts" ICED 01, pp. 179-186, Glasgow, 21-23, 2001. 
7. Shanley F. R., (1960), Weight-strength Analysis of Aircraft Structures, $2^{\text {nd }}$ edn, New York, Dover.

8. Caldwell J.B. and Woodhead R.G., (1973), Ship structures: some possibilities for improvement, North East Cost Institution - Inst. Eng. \& shipbuilders - Transaction - vol 89, $101-120$.

9. Ashby M. F., (1991), "Materials and shape", Acta Metall. Mater., 39(6), 1025 - 1039.

10. Pasini D., (2006), Shape Transformers for Material and Shape Selection of Lightweight Beams, Journal of Materials and Design, in press.

11. Pasini D, Smith DJ, Burgess SC., (2003), Structural efficiency maps for beams subjected to bending, P. Instn Mech Engrs, Part L: Journal of Materials: Design and applications; 217(3), 207-220.

12. Pasini D., Material and Shape Selection for optimizing flexural vibrations in multilayered resonators, Journal of Microelectromechanical Systems, in press, 2006.

13. Engesser, F. (1889), Ueber die Knickfesrigkeit Gerader St ${ }^{\top M}$ be. Zeitschrift f) r Architektur und Ingenieurwesen, Vol 35, N 4 Hannover, reported in Timoshenko, S. P., (1953), History of strength of materials, New York, McGraw-Hill.

14. Karman, T. (1956), Collected woks of Theodore von Karman, Butterworths Scientific Publications, London.

15. Shanley, F. R. (1946), “The column paradox”, Journal of the Aeronautical Sciences, Vol 13, $\mathrm{N} 12$.

16. X. Guo, G.D. Cheng and N. Olhoff, (2005), "Optimum design of truss topology under buckling constraints”, Struct. Multidisc Optim vol 30, N. 3, 169-180.

17. Case, J., Chilever, L., Ross C.T.F., (1999), Strength of materials and structures, Arnold, London. 


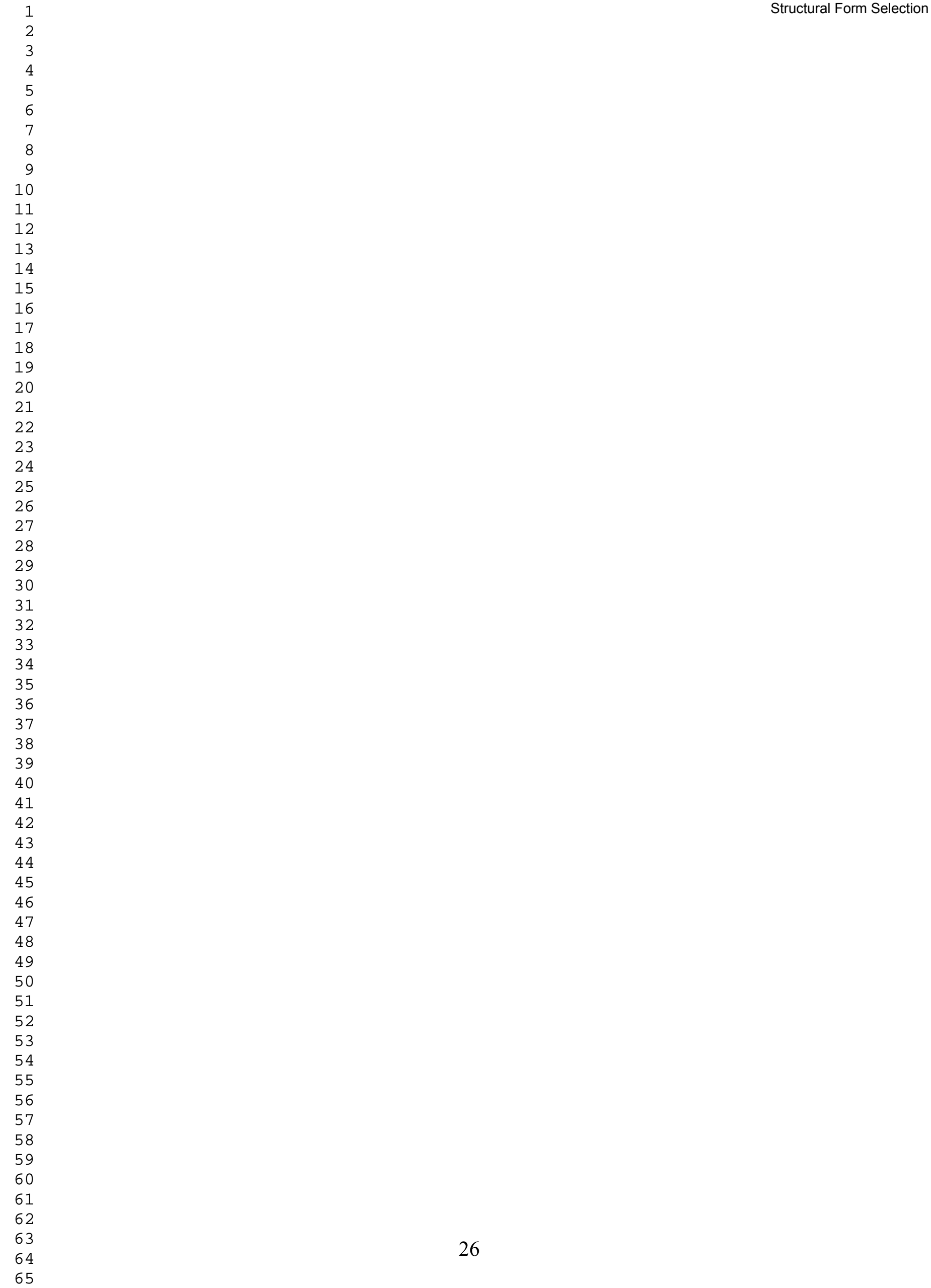




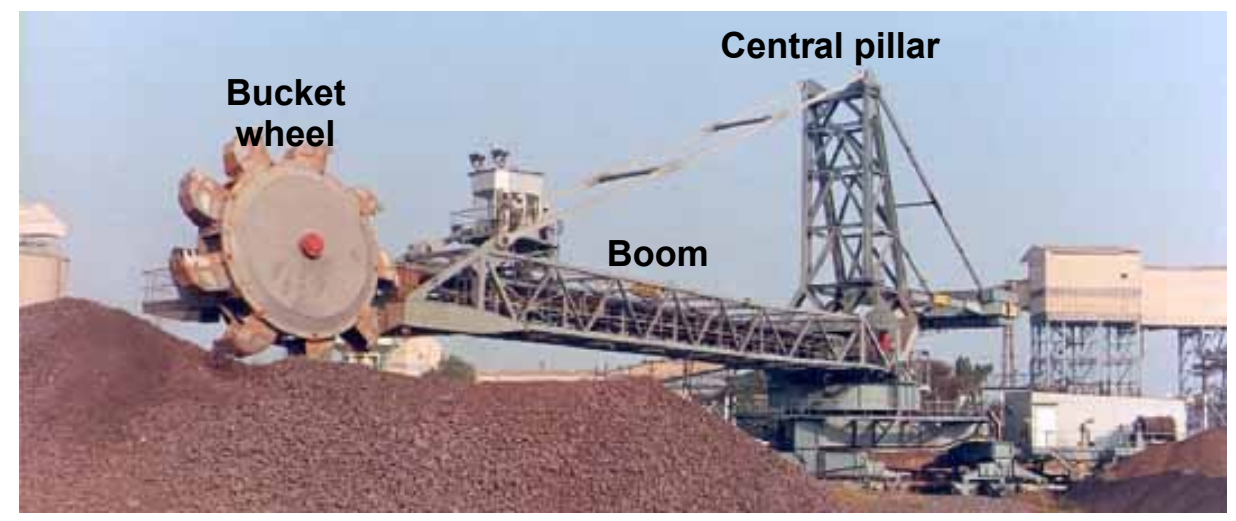

Figure 1. Handling machine for material reclaiming. 


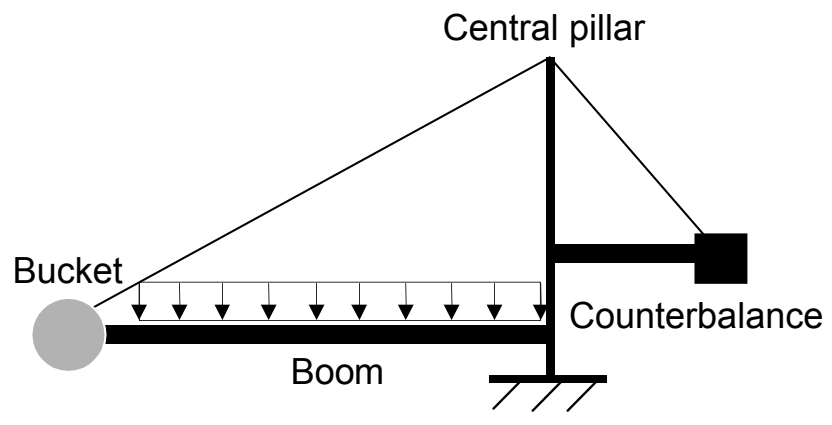

Figure 2 The structure cannot occupy the space where the conveyor belt is located, i.e. shadow region. 

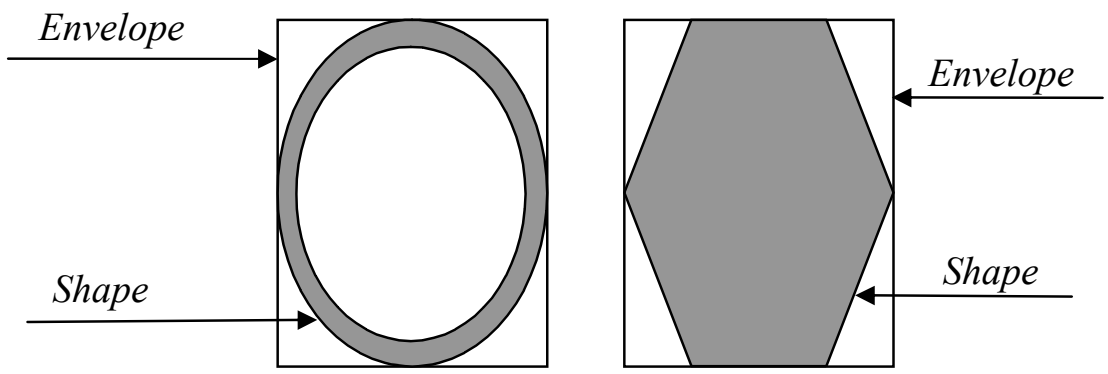

Figure 3. The constituents of a cross-section: the Envelope and the Shape. 

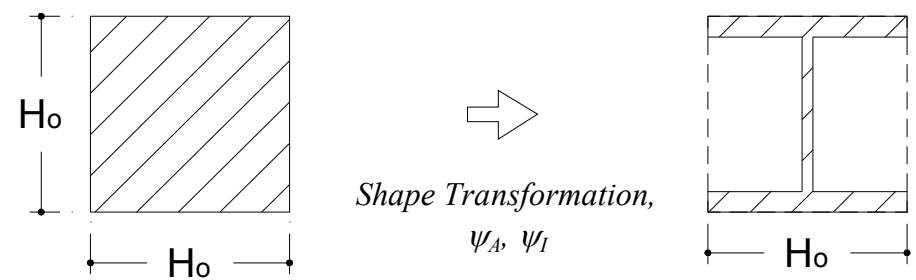

Envelope scaling,

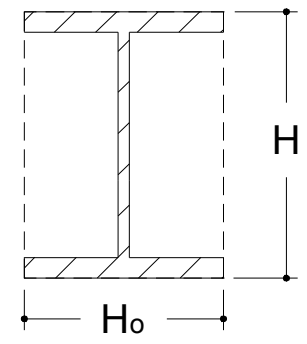

Figure 4 The shape transformation of a cross-section and its envelope scaling in the vertical direction. 

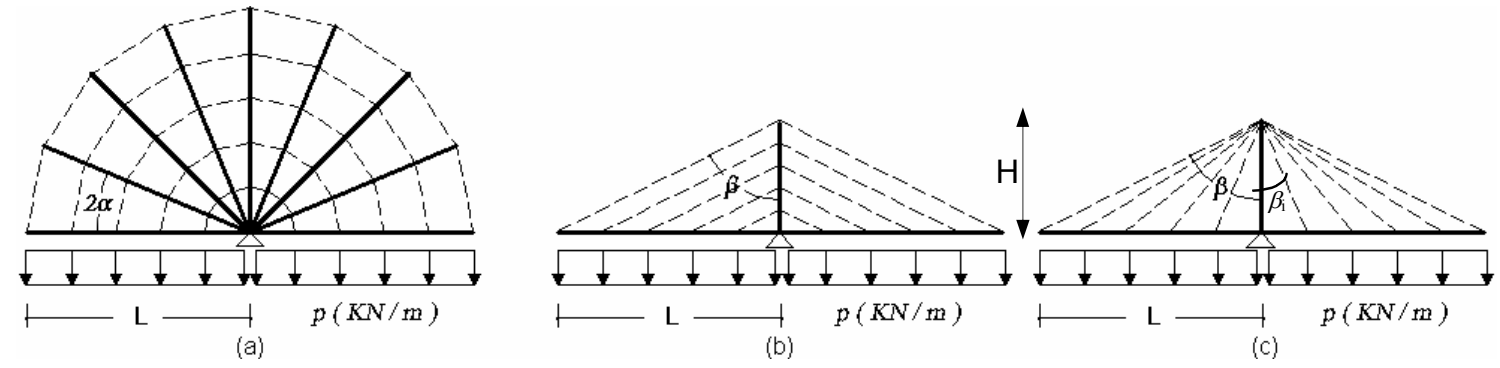

Figure 5 Three different structural forms: a) Web structure, b) Parallel structure and c) Convergent structure. (Note: dashed thin lines for tension members, continuous thick lines for compression and/or bending elements). 


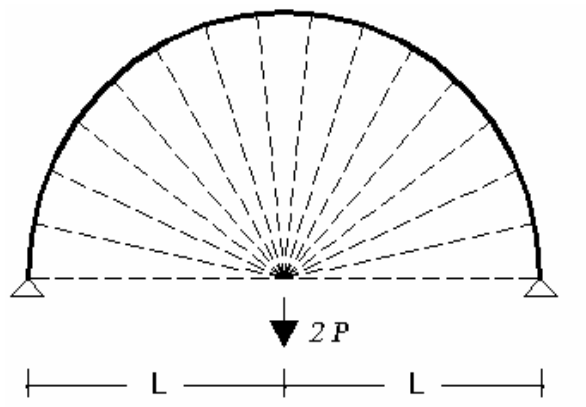

(a)

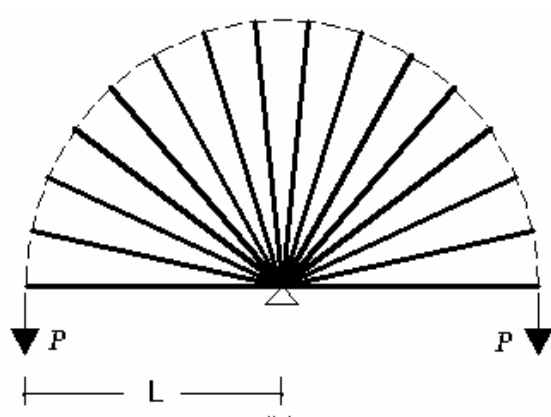

(b)

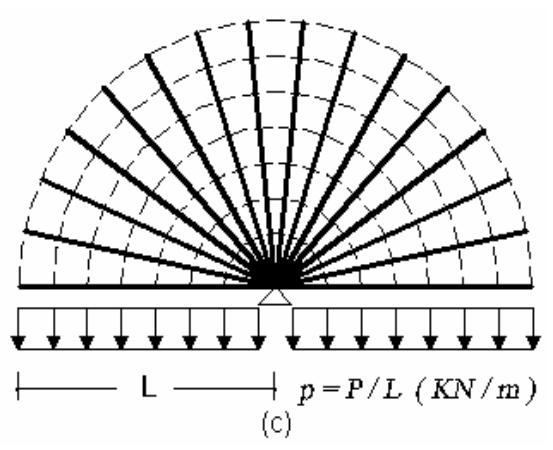

Figure 6 a) Michell structure for point central load, b) Inverted Michell structure for end point loads, c) derived Michell frame for uniform load. 


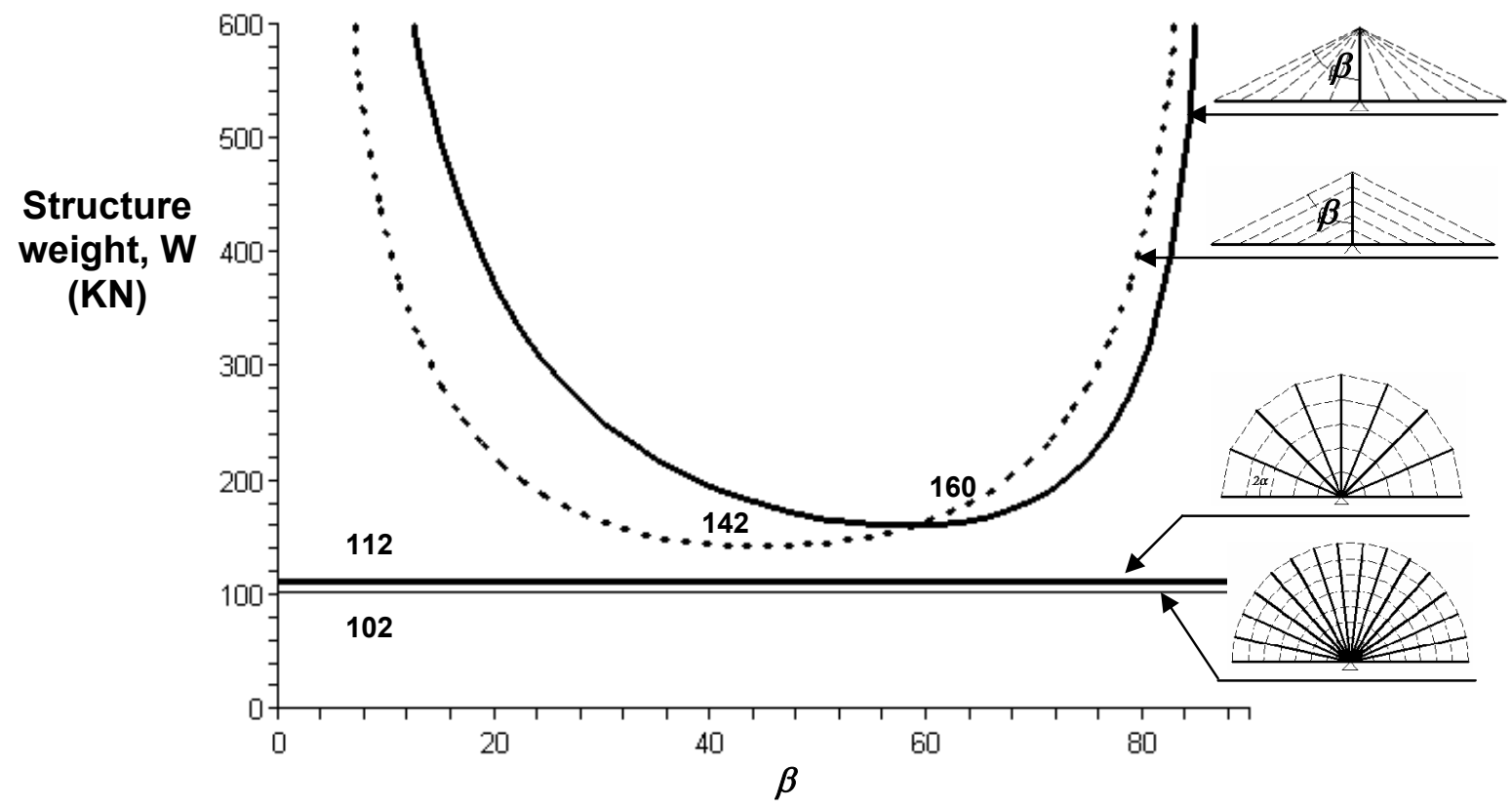

Figure 7 Structure weight as a function of the angle $\beta$. Yield is the failure mode. 
SHAPE CONTRIBUTION $\lambda=3 / 4$

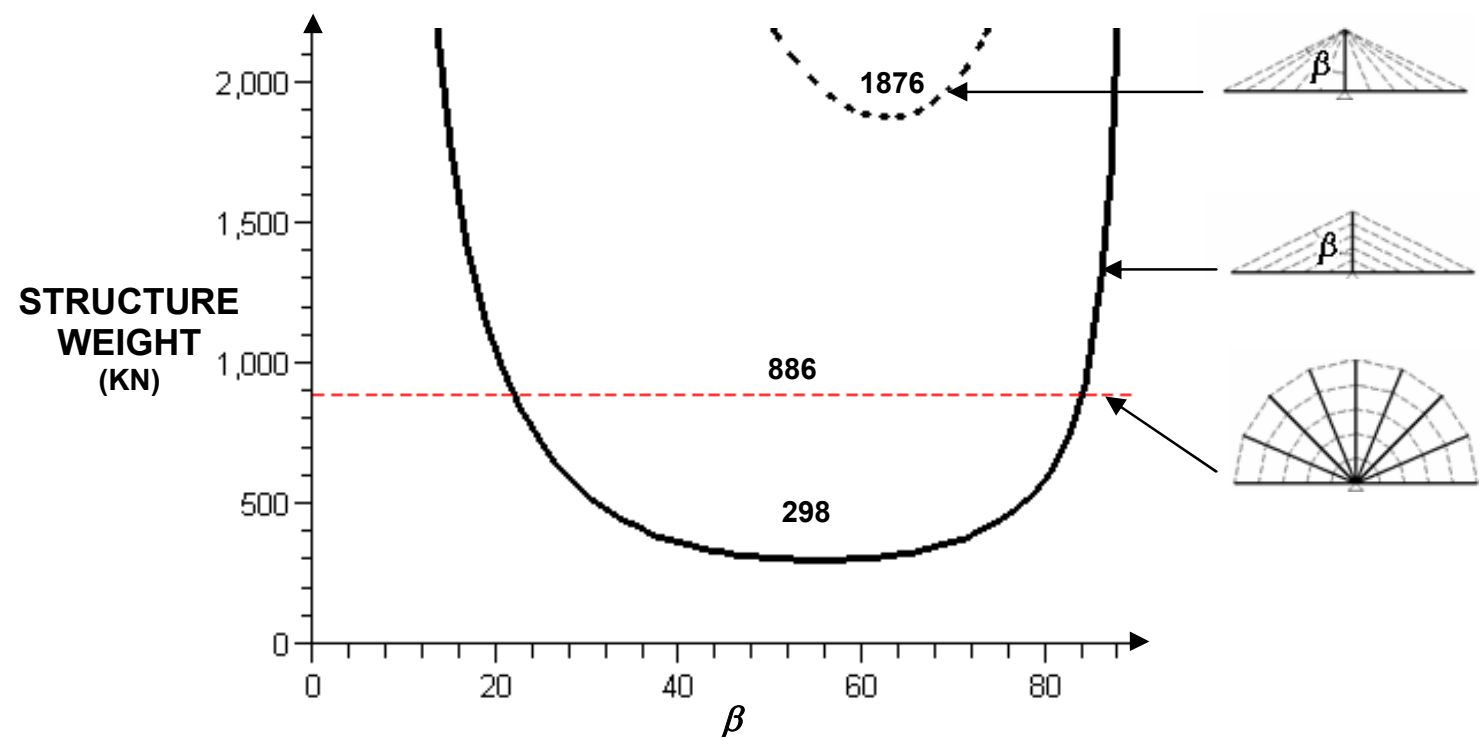

Figure 8 Weight as a function of the angle $\beta$ for solid circular sections 


\section{O}

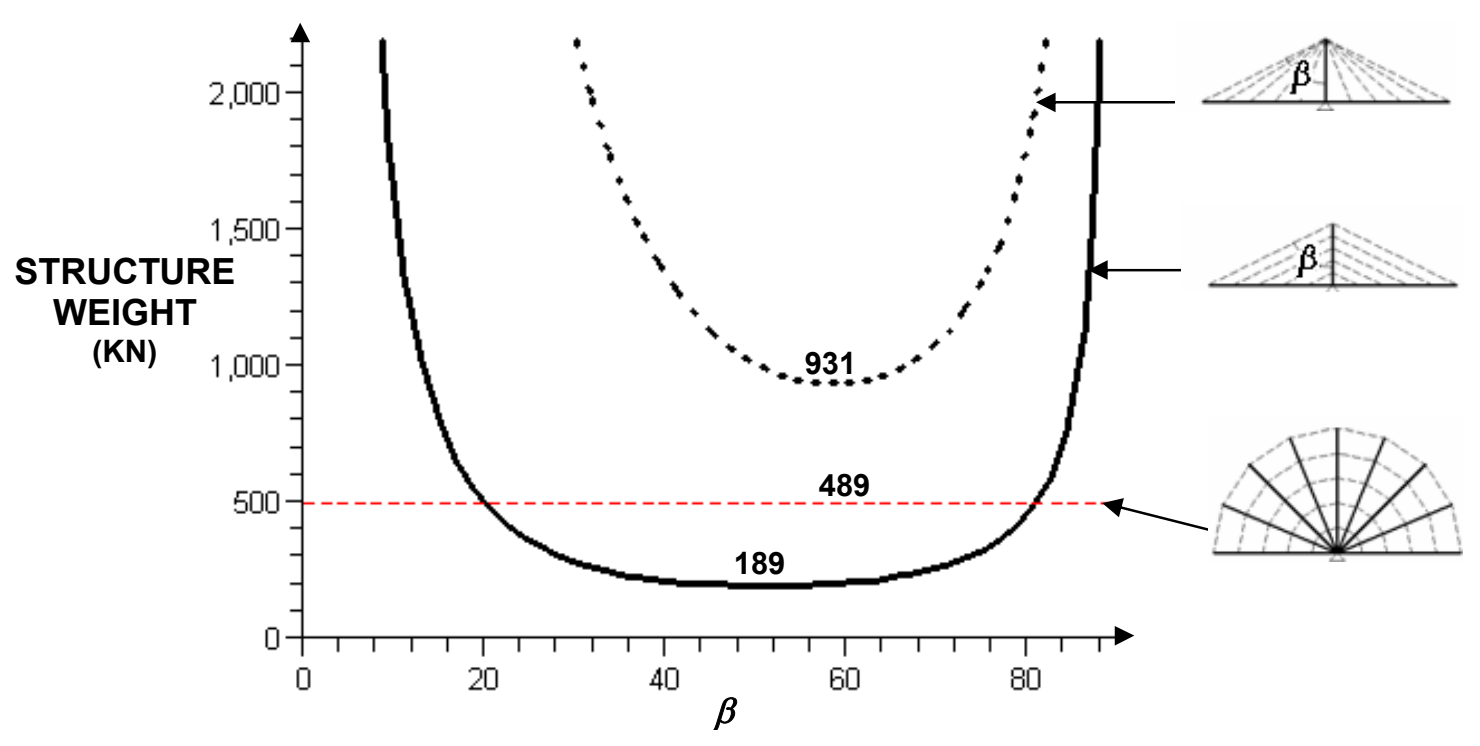

Figure 9 Weight as a function of the angle $\beta$ for circular hollow sections. 

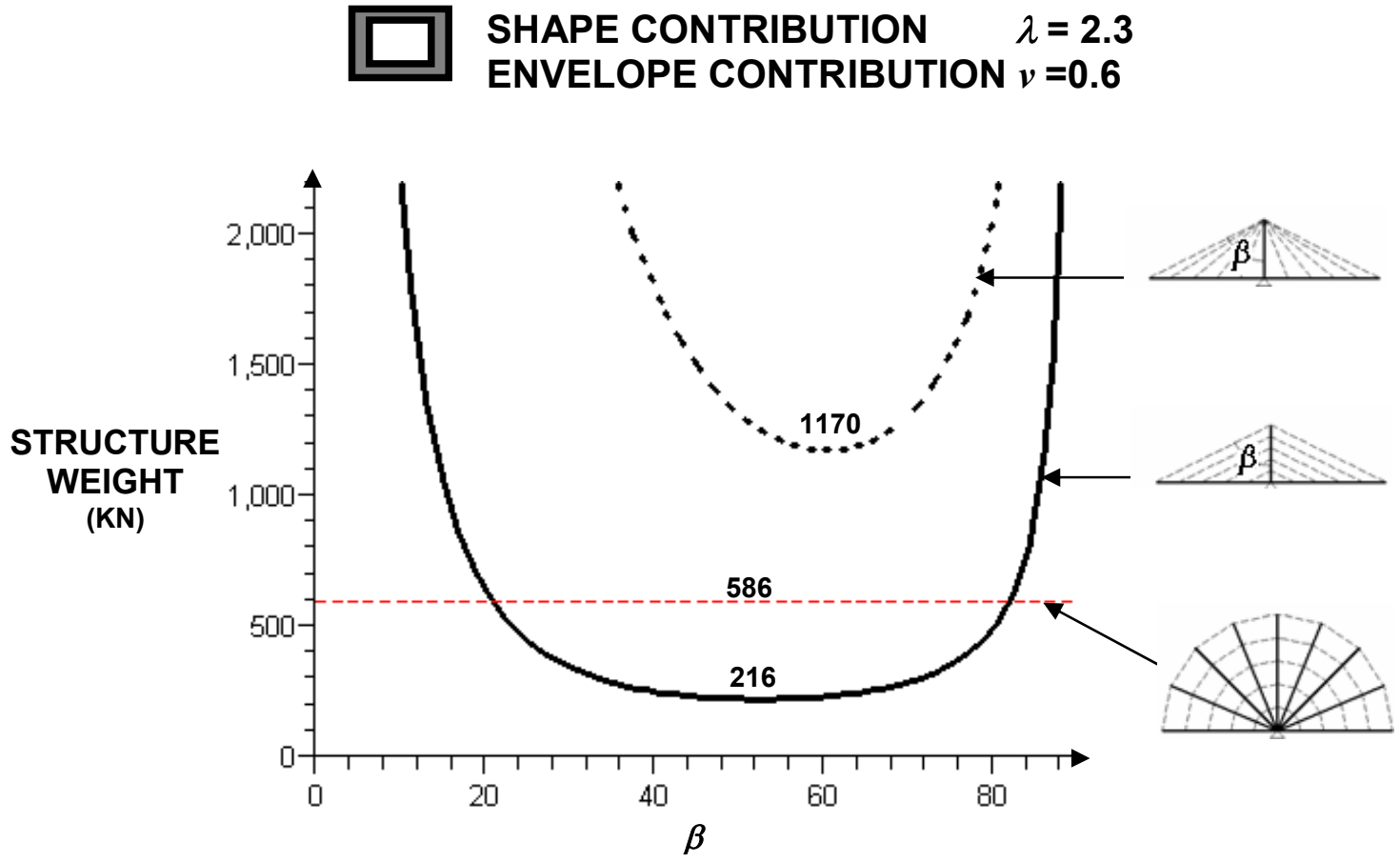

Figure 10 Weight as a function of the angle $\beta$ for hollow rectangular sections. 


\section{SHAPE CONTRIBUTION $\quad \lambda=\mathbf{2 . 3}$}

ENVELOPE CONTRIBUTION $v=1.1$

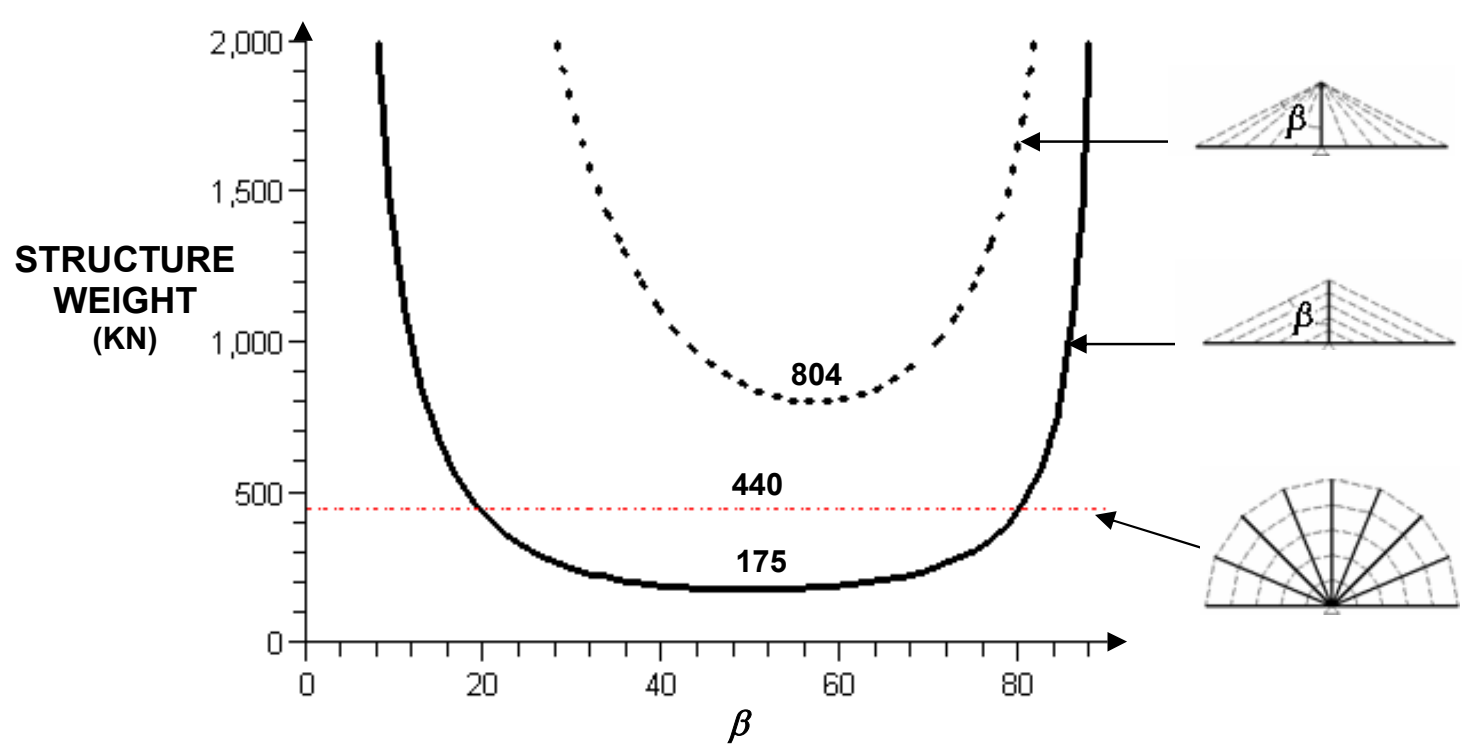

Figure 11 Weight as a function of the angle $\beta$ for hollow rectangular sections. 


\begin{tabular}{c|cc} 
Cross-section & $A$ & $I_{x x}$ \\
\hline & $B H$ & $\frac{B H^{3}}{12}$ \\
& $\frac{\pi}{4} B H$ & $\frac{\pi}{64} B H^{3}$ \\
\hline
\end{tabular}

Table 1. Area, $A$, and second moment of area about xx, $I_{x x}$, of common cross-sections 


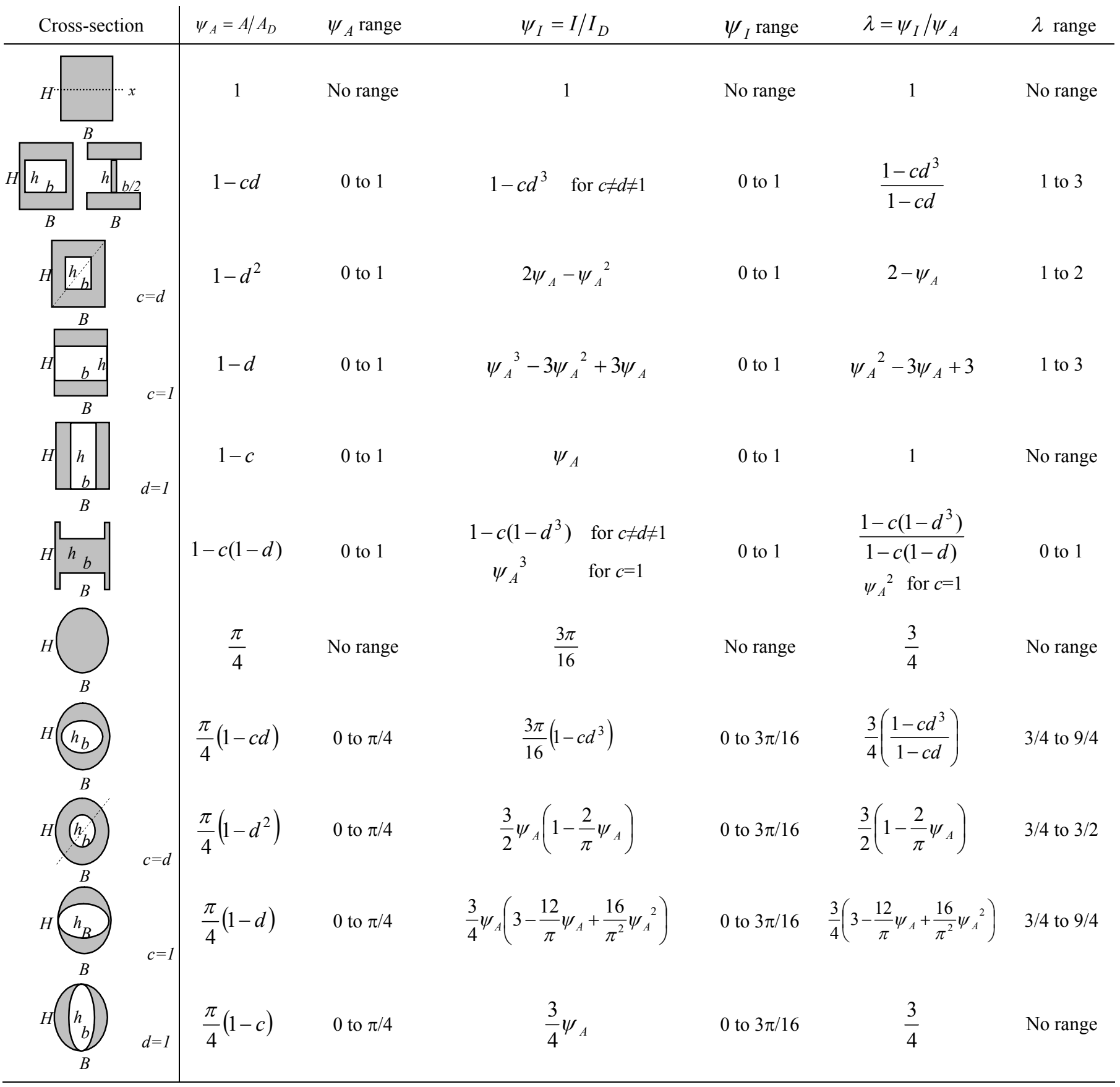

Table 2. Shape Transformers for the area and second moment of area of cross-sections with $c=b / B$ and $d=h / H$ 